\title{
Patient Reported Outcomes from Sacroiliac Joint Fusion
}

\author{
Vamsi K. Kancherla ${ }^{1}$, Shane M. McGowan ${ }^{1}$, Brittany N. Audley ${ }^{2}$, Gbolabo Sokunbi $^{1}$, Steven T. Puccio ${ }^{1}$ \\ ${ }^{1}$ Department of Orthopaedic Surgery, St. Luke's University Health Network, Bethlehem, PA, USA \\ ${ }^{2}$ Temple/St. Luke's Medical School, Bethlehem, PA, USA
}

Study Design: Retrospective, case series.

Purpose: The purpose of this study is to determine morbidity, complications, and patient reported outcomes from minimally invasive sacroiliac joint (SIJ) fusion.

Overview of Literature: Lumbar back pain emanating from the SIJ can be surgically treated via a percutaneous approach in the appropriately selected patient with minimal morbidity and acceptable functional outcomes.

Methods: Patients diagnosed by $>2$ physical examination maneuvers and subjective relief from a computed tomography-guided lidocaine-bupivacaine-steroid injection underwent SIJ fusion after failing conservative management with a combination of oral antiinflammatory medications, physical therapy, and pelvic belt stabilization. Perioperative data collected include estimated blood loss (EBL) and operative time. Oswestry disability index, 12-item short form health survey (SF-12), visual analogue score, and functional status were analyzed. All complications were noted.

Results: The study cohort of 45 cases (69\% female) achieved postoperative survey follow-up at 9.9 and 32.3 months. SF-12 physical component summary statistically improved while all other scores were equivalent. Mean EBL and operative time were $22 \mathrm{~mL}$ and 36 minutes, respectively. Initial survey showed that $64 \%$ of patients discontinued narcotics (29/45), $71 \%$ do not use an assistive device (32/45), and $15.6 \%$ do not work due to pain (7/45). $73 \%$ of patients stated they would have the surgery again (33/45). For the second survey, $65 \%$ of patients discontinued narcotics (26/40), $70 \%$ did not use an assistive device (28/40), and $17.5 \%$ did not work due to pain (7/40). A history of thoracolumbar instrumentation (16/45) did not significantly affect outcomes. Three complications described by screw malposition with neurologic deficit (6.7\%) were treated with screw repositioning (1 case) and removal of a single superior implant ( 2 cases) with time to revision of 2.2 months. All three ultimately had resolution of radicular pain.

Conclusions: Percutaneous SIJ fusion offers minimal morbidity and acceptable functional outcomes. While women and those with a prior history of lumbar instrumentation may be at increased risk of having SIJ dysfunction requiring surgical intervention, it was not found to affect postoperative functional outcomes when compared to the non-instrumented group.

Keywords: Sacroiliac; Fusion; Morbidity; Outcomes

\section{Introduction}

Pain localized around the low back, groin, and gluteal area can originate from the lumbar spine and hip joint. From a historical standpoint, in the early 1900s the sacroiliac joint (SIJ) as a source of pain had lost traction as diagnostic mo-

\footnotetext{
Received Mar 25, 2016; Revised Jun 2, 2016; Accepted Jun 17, 2016

Corresponding author: Vamsi K. Kancherla

Department of Orthopaedic Surgery, St. Luke's University Health Network,

801 Ostrum St, PPHP2, Bethlehem, PA 18015, USA

Tel: +847-809-1118, Fax: +484-526-1593, E-mail: kanchev@ccf.org
} 
dalities were better able to definitively identify discogenic pain, radicular pain, herniated discs, and facet arthropathy among others as true etiologies for pain. Patients were unfortunately often found to have pain, dysfunction, and instability despite having treatment (operative and nonoperative) for the aforementioned diagnoses. Pain at the SIJ can be idiopathic, degenerative, post-traumatic, postinfection, and/or due to adjacent segment disease after lumbar fusion surgery [1-16]. There is also some evidence to suggest a correlation between leg length discrepancy, SI joint instability and subsequent pain $[17,18]$.

The SIJ is a site often overlooked as a pain generator in patients presenting with back pain. SIJ pain is suggested as the main culprit in as many as $22 \%$ of patients presenting for evaluation of acute and chronic low back pain [2]. Historically, arthrodesis of this joint has been addressed via large open arthrodesis techniques. Open techniques are associated with increased blood loss, risk of infection, longer hospitalizations and longer recovery periods [2]. Newer minimally invasive techniques have since emerged that allow for a means to address this challenging diagnosis while minimizing morbidity $[2,3,7,14]$. In patients who have failed conservative management in the way of nonsteroidal anti-inflammatory drugs (NSAIDs), physical therapy, pelvic belt stabilization, and in whom image guided joint injections have demonstrated a positive response, operative treatment with minimally invasive arthrodesis may be a definitive solution. Several studies have shown excellent functional outcomes and resolution of symptoms, however many of them are vendor sponsored with potential for conflicts of interest [2,3,7,14,19-22]. We hypothesize that a less invasive SIJ fusion technique may lead to favorable clinical outcomes with minimal associated surgical morbidity provided all other reasons for back pain have been adequately ruled out.

\section{Materials and Methods}

A retrospective analysis identifying all patients undergoing percutaneous SIJ fusion by a single surgeon between March 2012 and March 2014 was performed at our institution. The patients were selected based on history, physical exam and a single computed tomography (CT) guided lidocaine-bupivacaine-steroid injection. A positive result on 3 or more pain provocation tests (Fortin finger test, Gaenselen's maneuver, flexion abduction external rotation, compression, distraction, thigh thrust) and subjec- tive improvement from an intra-articular injection were sufficient for diagnosis. All patients were recalcitrant to non-operative treatment which included some combination of activity modification, NSAIDs, physical therapy, and pelvic belt stabilization. Anteroposterior pelvis and lumbar spine X-rays were performed on all patients to help rule out any other etiologies for back pain. Advanced imaging in the form of magnetic resonance imaging was performed if there was concern for an alternative etiology.

Patients were excluded if they were less than 18 years old, infected, had previous SIJ surgery, or if there was suspicion for an alternative etiology for back pain. Sixty-one cases (57 patients) were identified and underwent fusion. 47 cases (43 patients) had available operative reports, radiographs, inpatient notes, and outpatient notes. Of these, 45 cases (41 patients) were available for outcomes assessment. All cases were reviewed to obtain demographic data as well as medical and surgical history. All patients had $\mathrm{X}$-rays at follow-up and only those with complications required additional imaging.

The surgical technique and steps were as follows. Preoperative skin marking was determined with fluoroscopy to be in line ( $3 \mathrm{~cm}$ in length) with the posterior cortex of the sacral body below the sacral ala. After subcutaneous dissection, guide pins were advanced from lateral to medial under fluoroscopy to assure appropriate position on lateral (below alar line, middle $1 / 3$ of the first sacral body), inlet (middle to anterior 1/3 sacral body), and outlet (parallel to S1 endplate without foraminal breach) views. After determining the implant length, each of the pins were overdrilled, broached, and secured with the chosen implant. Prior to closure, the wounds were irrigated and FloSeal hemostatic agent was used to control for any potential bleeding.

Primary outcome scores via a follow-up survey included Oswestry disability index (ODI), 12-item short form health survey (SF-12) with physical component summary (PCS) and mental component summary (MCS) scores, and visual analogue score (VAS). Surgical morbidity was ascertained from estimated blood loss (EBL), operative time, and length of stay (LOS). Secondary outcomes focused on narcotic use, return to work, use of assistive devices, influence of prior thoracolumbar surgery on outcomes, and desire to have surgery again. All complications requiring revision surgery were also recorded. Statistics utilizing a Student's $t$-test were performed using SPSS ver. 21 (IBM Corp., Armonk, NY, USA). 


\section{Results}

A total of 45 cases with an average age of 52.7 years and body mass index of 28.6 were reviewed (Table 1): 14 male, 31 female. There were 22 right sided and 23 left sided fusions. Two different implant systems were used; 36 performed via the iFuse SI Joint Fusion System (iFuse system, SI-BONE Inc., San Jose, CA, USA) and 9 performed via the SAMBA Screw System (SAMBA System, Medical Designs, LLC, Sioux Falls, SD, USA).

Women were found to have higher SF-12 MCS scores $(p<0.05)$. A history of smoking was found in 9 cases $(20 \%)$ while that of thoracolumbar surgery with instrumentation

Table 1. Patient demographics

\begin{tabular}{lc} 
Variable & Value \\
No. of cases & 45 \\
\hline Age $(\mathrm{yr})$ & $52.7 \pm 12.1(33.3-84.5)$ \\
\hline Body mass index $\left(\mathrm{kg} / \mathrm{m}^{2}\right)$ & $28.6 \pm 5.2(19.7-45.5)$ \\
Sex (male:female) & $14: 31$ \\
Operative side (right:left) & $22: 23$ \\
Implant (iFuse Sl Bone: Samba Screw) & $36: 9$ \\
\hline History of smoking & 9 \\
\hline History of thoracolumbar surgery & 16 \\
\hline
\end{tabular}

was found in 16 cases (36\%). Patients who smoked were found to have significantly lower SF-12 PCS and higher ODI scores $(p<0.05)$. In those with previous lumbar instrumentation, the distal most level included S1 in 11 cases, L5 in 4 cases, and L4 in 1 case. A history of thoracolumbar surgery with instrumentation did not significantly affect primary or secondary outcomes (Table 2). There were 4 patients that ultimately required contralateral fusion, accounting for 8 of the 45 total cases. 2 of these patients had a history of thoracolumbar surgery, 1 of which had instrumentation to L5. Contralateral fusion surgery occurred at a mean of 4.9 months (standard deviation [SD], 3.6; $R, 1.4-9.4$ ) from index surgery. Outcomes scores in this bilateral group were not found to be different from the overall cohort.

The mean EBL was $22 \mathrm{~mL}$ (SD, 33; range, 0-150) and the operative time was 36 minutes (SD, 12; range, 22-81). While clinical follow up ended at 6.7 months, survey follow up was performed at 9.9 months (SD, 6.1; range, 0.7-21.9) and 32.6 months (SD, 6.3; range, 21.1-43.8). Initial survey determined the following scores: SF-12 PCS 35.9, SF-12 MCS 47.8, and VAS 4.6. VAS was significantly improved from preoperative VAS 8.7 (SD, 1.4; range, 4-10; $p<0.0001$ ). Subsequent, $>2.5$ year repeat survey follow-up found improved SF-12 PCS 40.2 ( $p=0.04)$ and equivalent remaining scores (Table 3). The average LOS was 0.42 days with 29/45 staying 0 days, $13 / 45$ staying 1 day, and $3 / 45$ staying 2

Table 2. Effect of sex, instrumentation, and smoking on outcomes: $p$-values

\begin{tabular}{lcccccc} 
Variable & $\begin{array}{c}\text { SF-12 } \\
\text { PCS }\end{array}$ & $\begin{array}{c}\text { SF-12 } \\
\text { MCS }\end{array}$ & ODI & $\begin{array}{c}\text { Preoperative } \\
\text { VAS }\end{array}$ & $\begin{array}{c}\text { Postoperative } \\
\text { VAS }\end{array}$ & $\begin{array}{c}\text { Desire for } \\
\text { surgery again }\end{array}$ \\
Female vs. male & 0.72 & 0.05 & 0.39 & 0.32 & 0.83 & 0.40 \\
Instrumentation vs. none & 0.37 & 0.90 & 0.29 & 0.52 & 0.09 & 0.63 \\
Smoking vs. no smoking & 0.03 & 0.26 & 0.04 & 0.46 & 0.09 & 0.08 \\
\hline
\end{tabular}

SF-12, 12-item short form health survey (SF-12); PCS, physical component summary; MCS, mental component summary; ODI, Oswestry disability index; VAS, visual analogue score.

Table 3. Primary outcomes

\begin{tabular}{lccc} 
Variable & Early survey follow-up & Late survey follow-up & $p$-value \\
No. of cases & 45 & 40 & - \\
SF-12 PCS & $35.9 \pm 10.6(16.9-57.2)$ & $40.2 \pm 8.7(25.1-55.5)$ & 0.04 \\
\hline SF-12 MCS & $47.8 \pm 11.4(19-66.3)$ & $45.4 \pm 8.7(25.6-59.8)$ & 0.27 \\
ODI & $37.4 \pm 19.3(0-74)$ & $35.2 \pm 17.8(0-68)$ & 0.57 \\
VAS & $4.6 \pm 2.8(1-10)$ & $4.0 \pm 2.4(1-10)$ & 0.23 \\
\hline
\end{tabular}

SF-12, 12-item Short Form health survey (SF-12); PCS, physical component summary; MCS, mental component summary; ODI, Oswestry disability index; VAS, visual analogue score. 
Table 4. Secondary outcomes

\begin{tabular}{|c|c|c|}
\hline & Early & Late \\
\hline Still using narcotics (\%) & 38 & 35 \\
\hline Ambulating independently (\%) & 29 & 30 \\
\hline Desire to have surgery again (\%) & 73 & 70 \\
\hline Return to work time & \multicolumn{2}{|c|}{$\begin{array}{l}<1 \text { week (1), <1 month (6), 1-2 months (7), 2-3 months (2), 6-12 months (1), do not work (18), } \\
\text { pain prevents ability to work (5), retired (2), disabled (2), workmens compensation (1) }\end{array}$} \\
\hline Complications & \multicolumn{2}{|c|}{$\begin{array}{l}3 \text { malpositioned screws with neurologic deficit treated with repositioning (1) and removal } \\
\text { of superior implant (2) }\end{array}$} \\
\hline Time to revision (mo) & \multicolumn{2}{|c|}{$2.2 \pm 2.1(0-4.2)$} \\
\hline
\end{tabular}

days. Sixty-four percent of patients stopped using narcotics (29/45), 71\% no longer use an assistive device (32/45), and $15.6 \%$ did not return to work due to pain (7/45). Seventy-three percent of patients stated they would have the surgery again (33/45) (Table 4). These secondary outcomes were maintained at later follow up.

A total of 3 complications described by CT confirming screw mal-positioning with neurologic deficit (Table 4) were noted $(6.7 \%)$ and were treated with screw repositioning ( 1 case: SambA Screw) and removal of a single superior implant ( 2 cases: 1 Samba Screw and 1 iFuse SI Bone Implant) with an average time to revision of 2.2 months (SD, 2.1). 2 of these patients continue to have moderate gluteal pain while the remaining 1 patient achieved acceptable pain relief. All three ultimately had resolution of radicular pain and restoration of neurologic function.

\section{Discussion}

SIJ pain may account for $15 \%-30 \%$ of non-radicular low back pain [1]. From an economic standpoint, nonoperative treatment of SIJ disruption is associated with substantial costs and medical resource utilization for both commercial payer and Medicare populations [23,24]. Recent evidence suggests that minimally invasive surgery may be advantageous in reducing the economic burden [25]. Though the management of SIJ pain can be complex, an understanding of the relationship between the sacrum, ilium, and surrounding soft tissues can help identify the etiology and guide appropriate treatment.

The SIJ is a diarthrodial joint with a mean surface area of $17.5 \mathrm{~cm}^{2}$ and consists of two surfaces held together by fibrous capsule and enjoined with synovial fluid [1]. The soft tissues (ligaments, muscles, and fascia) surrounding the joint serve primarily to support the upper body and reduce the impact of ambulation. Motion at this joint is minimal with mean rotation and translation ranging from $1^{\circ}-12^{\circ}$ and $3-16 \mathrm{~mm}$, respectively. The innervation of the posterior joint has been described to arise from the dorsal rami of L5-S4 in most individuals while that of the ventral joint arises from the ventral rami of L4-S2 [1].

Many patients often identify the area of the buttocks and posterolateral thigh as the source of pain with the worst area often located within $10 \mathrm{~mm}$ of the posterior superior iliac spine [1]. This off-midline pain typically follows an inciting event in $40 \%-50 \%$ of patients [1]. From a physical examination standpoint, Laslett et al. [26] found the presence of three of six provocation tests to have $94 \%$ sensitivity and $78 \%$ specificity in predicting a positive response to a single diagnostic SIJ injection. A systematic review by Szadek et al. [15] concluded that three positive provocation tests had significant discriminative power (diagnostic odds ratio, 17.16) for diagnosing SIJ pain using the reference standard of two positive blocks. When looking at a guided block alone, Polly et al. [27] found a $50 \%$ reduction in pain to correlate well with excellent postoperative outcomes.

Previously described etiologies for pain generation have included leg length discrepancy (LLD), transitional anatomy, gait and biomechanical abnormalities, trauma, infection, scoliosis, pregnancy, and history of spine surgery [1]. LLD can often result in pelvic obliquity that leads to subsequent mechanical mal-alignment of the SIJ resulting in high loads passing through the joint [18]. A finite element analysis by Kiapour et al. [18] in 2012 found the load and the peak stresses across the SIJ articular surfaces progressively increased with an increase in LLD. Clinical studies have supported this finding by showing $>5 \mathrm{~mm}$ of LLD to be sufficient to cause pain [17]. Pregnancy can result in SIJ pain by virtue of weight gain, exaggerated 
lordotic posture, third-trimester hormone-induced ligamentous relaxation and the pelvic trauma associated with parturition [1]. The roughly 2:1 female predominance in our cohort may suggest some underlying hormonal reasons (hyperlaxity) for SIJ pathology, however future larger power studies may help delineate this further. None of the patients had a history of pelvic trauma, infection, transitional anatomy, or recent pregnancy.

Additionally, a significant amount of recent literature has brought attention to the presence of lumbosacral fusion constructs as a risk factor for SIJ pain based on the notion of adjacent segment disease [4,5,8,10-13,16,28]. Ivanov et al. [5] used simulated surgical procedures and a finite elemental spine-pelvis model to assess angular motion and stress across the SIJ following spinal fusion. They found increased SIJ stress after surgery, which was least following L4-5 fusion and greatest after L4-S1 fusion $[1,5]$. A sub-analysis of our cohort found that $36 \%$ of patients had an instrumented thoracolumbar spine; however, it did not significantly influence primary or secondary outcomes from fusion when compared to the uninstrumented patients. Additionally, of the 4 patients that ultimately required contralateral SIJ fusion at a mean of 4.9 months from index fusion, only 1 patient had a history of thoracolumbar instrumentation (down to L5).

The morbidity associated with an open surgical intervention to the SIJ has been met with a recent interest in minimally invasive, percutaneous SIJ fusion. A recent multi-center study by Smith et al. [14] comparing the open approach to a less invasive approach found significant improvement in VAS pain ratings in both groups and the less invasive approach to have significantly improved EBL ( $288 \mathrm{~mL}$ vs. $33 \mathrm{~mL}$ ), operative time (163 minutes vs. 70 minutes), and length of hospitalization (5.1 days vs. 1.3 days). Ledonio et al. [7] in 2014, further substantiated these findings and also found ODI scores to be no different between groups. Similarly, our cohort demonstrated favorable morbidity with EBL, operative time, and LOS as well as clinical scores to that of current literature. Nevertheless, there continue to be patients who do not obtain acceptable results. We suggest that surgeons place emphasis on an accurate diagnosis prior to surgical intervention and have a detailed discussion with the patient preoperatively to ensure they understand that there is a fair chance of a suboptimal outcome. Failure to obtain relief may be due to incorrect indications, lack of biologic fusion, and/ or presence of co-pathologies.
Prior studies looking at more open, graft stabilized SIJ documented complication and revisions rates as high as $30 \%$ and $15 \%$, respectively [14]. SIJ fusion via a less invasive method has been shown to be safer and more effective [3,29]. Miller et al. [19] in 2013 found the complication and revision rates to be $3.8 \%$ and $1.8 \%$, respectively at a median follow-up of 4 months. The most common complaints in order of frequency were pain $(2.2 \%)$, nerve impingement (0.9\%), and recurrent SIJ pain (0.8\%) [19]. Revisions were typically performed in the early postoperative setting for treatment of a symptomatic malpositioned implant $(0.9 \%)$ or to correct an improperly sized implant in an asymptomatic patient $(0.2 \%)$. Revision in the late postoperative setting was typically for symptom recurrence $(0.6 \%)$ and continued pain of undetermined etiology (0.1\%) [19]. At this point, no study has clinically evaluated adjacent segment disease to the lumbar segment but a finite element study has shown minimal $(<5 \%)$ increase in range of motion in the lumbar spine [30]. In our cohort, 3 patients $(6.7 \%)$ required revision surgery due to neurologic injury from a CT confirmed malpositioned implant. All 3 patients ultimately had resolution of neurologic deficits.

As with any surgical case involving instrumentation, close attention to detail is emphasized when analyzing sacral anatomy/morphology. A thorough understanding of each individual's anatomy must compliment the familiarity of the implants being utilized. Quality of imaging preoperatively and intraoperatively must be optimized to minimize poor visualization which in-turn could lead to malpositioning of implants. In specific, intraoperative lateral fluoroscopic views can help assure implant placement below the sacral ala while inlet/outlet pelvic views will confirm trajectory within the sacral body away from L5 root injury or foraminal breach. Additionally, for patients with concerning anatomy noted on preoperative X-rays, advanced imaging utilizing a CT scan with $3 \mathrm{D}$ reconstructions may be helpful in reducing potential complications. Factors such as body habitus, congenital anomalies (short or C-shaped sacrum) and prior surgeries may make placement of implants difficult, occasionally allowing only the placement of 2 proximal implants.

The limitations of this study include a small sample size and all those associated with a retrospective review. While we did obtain preoperative VAS scores, we did not have any other preoperative data for postoperative comparison. However, we were able to obtain further follow 
up functional scores. As well, 16 patients were excluded from the original 57 patients due to lack of data points, which could suggest selection bias. Lastly, the objective of this study was to demonstrate clinical outcomes based on a less invasive fusion procedure. Unfortunately, we are unable to document any evidence of radiographic fusion bed given the technique of surgery utilized. The ongrowth of bone onto the implants does not project nicely in any radiographic platform. We assume on postoperative follow up imaging that a fusion had taken place if there were no radiographic signs of loosening/loss of fixation (halos around the implants).

\section{Conclusions}

In conclusion, SIJ pain requiring fusion via percutaneous, minimally invasive surgery can be safely performed, offer minimal morbidity, improve VAS, and result in acceptable ODI and SF-12 scores. Women were found to have higher SF-12 MCS scores and nonsmokers were found to have higher SF-12 PCS and ODI scores. A history of thoracolumbar surgery was not found to influence fusion outcomes. While many patients reduced their narcotic intake, discontinued assistive device use, returned to work, and expressed desire to have the surgery again, there continue to be patients who do not achieve acceptable results. This series suggests that a thorough workup with strict indications is paramount in achieving good functional outcomes with this technique. Ultimately, a significant number of patients may have suboptimal outcomes and this must be taken into consideration when counseling patients regarding operative intervention.

\section{Conflict of Interest}

No potential conflict of interest relevant to this article was reported.

\section{References}

1. Cohen SP, Chen Y, Neufeld NJ. Sacroiliac joint pain: a comprehensive review of epidemiology, diagnosis and treatment. Expert Rev Neurother 2013;13:99116.

2. Cummings J Jr, Capobianco RA. Minimally invasive sacroiliac joint fusion: one-year outcomes in $18 \mathrm{pa}-$ tients. Ann Surg Innov Res 2013;7:12.
3. Duhon BS, Cher DJ, Wine KD, Lockstadt H, Kovalsky D, Soo CL. Safety and 6-month effectiveness of minimally invasive sacroiliac joint fusion: a prospective study. Med Devices (Auckl) 2013;6:219-29.

4. Ha KY, Lee JS, Kim KW. Degeneration of sacroiliac joint after instrumented lumbar or lumbosacral fusion: a prospective cohort study over five-year followup. Spine (Phila Pa 1976) 2008;33:1192-8.

5. Ivanov AA, Kiapour A, Ebraheim NA, Goel V. Lumbar fusion leads to increases in angular motion and stress across sacroiliac joint: a finite element study. Spine (Phila Pa 1976) 2009;34:E162-9.

6. Kim JT, Rudolf LM, Glaser JA. Outcome of percutaneous sacroiliac joint fixation with porous plasmacoated triangular titanium implants: an independent review. Open Orthop J 2013;7:51-6.

7. Ledonio CG, Polly DW Jr, Swiontkowski MF. Minimally invasive versus open sacroiliac joint fusion: are they similarly safe and effective? Clin Orthop Relat Res 2014;472:1831-8.

8. Liliang PC, Lu K, Liang CL, Tsai YD, Wang KW, Chen HJ. Sacroiliac joint pain after lumbar and lumbosacral fusion: findings using dual sacroiliac joint blocks. Pain Med 2011;12:565-70.

9. Mason LW, Chopra I, Mohanty K. The percutaneous stabilisation of the sacroiliac joint with hollow modular anchorage screws: a prospective outcome study. Eur Spine J 2013;22:2325-31.

10. Park P, Garton HJ, Gala VC, Hoff JT, McGillicuddy JE. Adjacent segment disease after lumbar or lumbosacral fusion: review of the literature. Spine (Phila Pa 1976) 2004;29:1938-44.

11. Rudolf L. MIS Fusion of the SI joint: does prior lumbar spinal fusion affect patient outcomes? Open Orthop J 2013;7:163-8.

12. Schroeder JE, Cunningham ME, Ross T, BoachieAdjei O. Early results of sacro-iliac joint fixation following long fusion to the sacrum in adult spine deformity. HSS J 2014;10:30-5.

13. Slinkard N, Agel J, Swiontkowski MF. Documentation of outcomes for sacroiliac joint fusion: does prior spinal fusion influence the outcome? Eur Spine J 2013;22:2318-24.

14. Smith AG, Capobianco R, Cher D, et al. Open versus minimally invasive sacroiliac joint fusion: a multicenter comparison of perioperative measures and clinical outcomes. Ann Surg Innov Res 2013;7:14. 
15. Szadek KM, van der Wurff P, van Tulder MW, Zuurmond WW, Perez RS. Diagnostic validity of criteria for sacroiliac joint pain: a systematic review. J Pain 2009;10:354-68.

16. Yoshihara H. Sacroiliac joint pain after lumbar/ lumbosacral fusion: current knowledge. Eur Spine J 2012;21:1788-96.

17. Friberg O. Clinical symptoms and biomechanics of lumbar spine and hip joint in leg length inequality. Spine (Phila Pa 1976) 1983;8:643-51.

18. Kiapour A, Abdelgawad AA, Goel VK, Souccar A, Terai T, Ebraheim NA. Relationship between limb length discrepancy and load distribution across the sacroiliac joint: a finite element study. J Orthop Res 2012;30:1577-80.

19. Miller LE, Reckling WC, Block JE. Analysis of postmarket complaints database for the iFuse SI Joint Fusion System(R): a minimally invasive treatment for degenerative sacroiliitis and sacroiliac joint disruption. Med Devices (Auckl) 2013;6:77-84.

20. Cher DJ, Polly DW. Improvement in health state utility after sacroiliac joint fusion: comparison to normal populations. Global Spine J 2016;6:100-7.

21. Duhon BS, Cher DJ, Wine KD, Kovalsky DA, Lockstadt H. Triangular titanium implants for minimally invasive sacroiliac joint fusion: a prospective study. Global Spine J 2016;6:257-69.

22. Lingutla KK, Pollock R, Ahuja S. Sacroiliac joint fusion for low back pain: a systematic review and metaanalysis. Eur Spine J 2016;25:1924-31.

23. Ackerman SJ, Polly DW Jr, Knight T, Holt T, Cummings J. Management of sacroiliac joint disruption and degenerative sacroiliitis with nonoperative care is medical resource-intensive and costly in a United States commercial payer population. Clinicoecon Outcomes Res 2014;6:63-74.

24. Ackerman SJ, Polly DW Jr, Knight T, Holt T, Cummings J Jr. Nonoperative care to manage sacroiliac joint disruption and degenerative sacroiliitis: high costs and medical resource utilization in the United States Medicare population. J Neurosurg Spine 2014;20:354-63.

25. Cher DJ, Frasco MA, Arnold RJ, Polly DW. Costeffectiveness of minimally invasive sacroiliac joint fusion. Clinicoecon Outcomes Res 2016;8:1-14.

26. Laslett M, Aprill CN, McDonald B, Young SB. Diagnosis of sacroiliac joint pain: validity of individual provocation tests and composites of tests. Man Ther 2005;10:207-18.

27. Polly D, Cher D, Whang PG, Frank C, Sembrano J. Does level of response to SI joint block predict response to SI joint fusion? Int J Spine Surg 2016;10:4.

28. Unoki E, Abe E, Murai H, Kobayashi T, Abe T. Fusion of multiple segments can increase the incidence of sacroiliac joint pain after lumbar or lumbosacral fusion. Spine (Phila Pa 1976) 2016;41:999-1005.

29. Polly DW, Swofford J, Whang PG, et al. Two-year outcomes from a randomized controlled trial of minimally invasive sacroiliac joint fusion vs. nonsurgical management for sacroiliac joint dysfunction. Int J Spine Surg 2016;10:28.

30. Lindsey DP, Kiapour A, Yerby SA, Goel VK. Sacroiliac joint fusion minimally affects adjacent lumbar segment motion: a finite element study. Int J Spine Surg 2015;9:64. 